\title{
Sizing and Siting of Large-Scale Batteries in Transmission Grids to Optimize the Use of Renewables
}

\author{
Laura Fiorini, Giuliano Andrea Pagani, Member, IEEE, Paolo Pelacchi, Davide Poli, Member, IEEE, \\ and Marco Aiello, Senior Member, IEEE
}

\begin{abstract}
Power systems are a recent field of application of complex network research, which allows performing large-scale studies and evaluations. Based on this theory, a power grid is modeled as a weighted graph with several kinds of nodes and edges, and further analysis can help in investigating the behavior of the grid under critical conditions. Among the crucial aspects of a power network, those concerning flow limits and power flow distribution are gaining relevance due to the increasing introduction of large-scale renewable energy generation facilities. Storage systems are a key element in having a more sustainable but still reliable grid. This paper focuses on a new research challenge regarding the siting of the storage on the transmission grid and its appropriate sizing. The problem is tackled by considering realistic configurations based on the IEEE-RTS-96 bus and data coming from the Italian transmission operator, and evaluating novel economic and complex network-based metrics on these configurations. Power flows are modeled in a linear way, and the representative optimization problem is expressed as a linear programming problem. The results show the potential benefits of storage in transmission lines and indicate that the siting has a minor role in the optimal operation of the system.
\end{abstract}

Index Terms-Complex power grid, dc power flow, energy curtailment, flow-based centrality, power systems, renewable energy sources (RESs), RTS-96, storage.

\section{INTRODUCTION}

G IVEN their complexity and importance to modern economies, it is not surprising that power systems have received growing attention in the fields of network science and complex system analysis, especially with the aim to investigate the behavior of power grid in case of unexpected failure and of targeted attacks [1]-[4]. However, a pure topological approach might not catch most of the crucial aspects of a power network, such those concerning flow limits and power flow distribution through the grid [5], [6].

Manuscript received September 27, 2016; revised December 1, 2016; accepted January 4, 2017. This work was supported by the Netherlands Organization for Scientific Research through the NWO MERGE project under Contract 647.002.006. This paper was recommended by Guest Editor H. H.-C. Iu.

L. Fiorini, G. A. Pagani, and M. Aiello are with the Department of Distributed Systems, University of Groningen, 9747 AG Groningen, The Netherlands (e-mail: 1.fiorini@rug.nl; g.a.pagani@rug.nl; m.aiello@rug.nl).

P. Pelacchi and D. Poli are with the Department of Energy, Systems, Territory and Construction Engineering, University of Pisa, 56122 Pisa, Italy (e-mail: paolo.pelacchi@ing.unipi.it; davide.poli@unipi.it).
These features have become increasingly relevant as a result of fostering the use of renewable energy sources (RESs). In fact, the uncontrollable nature of renewable source generation poses new challenges to the network in terms of operational conditions: power flows do not follow any more the traditional paths from major centers of production to consumption ones, since most of RES plants are linked to the distribution grid or far away from consumption sites, e.g., off-shore wind farms. Therefore, the transmission network has to be ready to accommodate bidirectional flows and be flexible. The expansion and planning of power systems is, however, a long-lasting and expensive process that often has to face the strongest public opposition against new overhead lines [7], [8]. Storage technologies can be the key elements in the realization of an electrical system that is secure and reliable as the traditional one [9], [10], providing the (smart) flexibility required to increase the share of renewable to meet and exceed the goals of policy makers [11]-[13].

In this paper, the power systems are modeled as a weighted graph, taking into account flow limits, lines' reactance, and voltage angles, and four kinds of nodes are characterized according to the element they represent. The following research question is addressed: what is the optimal size and location of storage systems to increase the stability of the system, while reducing the operation costs? According to [14] and [15], batteries and underground CAES are the most attractive technologies for transmission and distribution grid support and load shifting. However, underground CAES requires geographical considerations that are out of the scope of this paper, while electrochemical batteries are more flexible [16], not only for mobility applications [17]. Therefore, this paper considers only large-scale batteries as storage technology.

To answer the above-mentioned research question, several configurations of sizing and siting of batteries are considered, using the high-voltage IEEE RTS-96 as test system, the Italian power system for modeling the energy demand, and a range of capacities and locations for wind and solar energy production. The configurations are seen as a graph on which power flows according to a linear model over subsequent time intervals. Flow and complex network-based metrics are used to model the physics of the system toward measures of economic optimality and system resilience. In particular, a novel application of betweennes is proposed. The analysis 
of the configurations shows that batteries are beneficial in storing energy in excess that otherwise would be curtailed; the siting of the storage has a limited influence on the optimality of the configuration. In addition, the batteries are beneficial in limiting the use of conventional power production plants during high demand periods using stored energy. The last aspect is important from an economic perspective showing a case for a large-scale adoption of batteries on the transmission network. In this paper, an investigation on how proper sizing (and to a smaller extent siting) of storage can enable a smarter use of the transmission grid allowing the flexibility required by a higher stake of renewables is presented. Policy and regulatory consideration are outside of the scope of this paper.

This paper is organized as follows. Section II provides an overview of the state of the art on the main ingredients of this paper: modeling the power systems as a graph, on the one hand, and the inclusion of storage in the electrical grid, on the other hand. Section III provides some fundamentals of power flows in HV-power systems and network theory, while Section IV defines the model used in this paper. Sections V and VI provide, respectively, the main features of the sizing and siting policies and the characteristics of the minimization problem to be solved. The details of the test case and the results are explained in Section VII. Concluding remarks and lines for future research are presented in Section VIII.

\section{State OF The ART}

A systemic approach is taken, and the transmission lines are modeled as a graph on which power flows. The time horizon covers a whole day, discretized in 15-min time intervals, and power flows are linearized. Batteries are the storage mean and are considered as nodes of the network [18]. This paper thus relates to the state of the art in network/graph models of power systems, on the one hand, and on storage systems for power transmission, on the other hand. As, to the best of the authors' knowledge, little work has been done on their combination, the state of the art in the two fields is reviewed separately, next.

\section{A. Complex Network Models for Power Systems}

Modeling power systems as a complex graph and looking at its statistics from a global perspective have been mostly the object of research assessing the reliability of the network, in particular to identify critical points [19], [20]. Rosas-Casals et al. [1] present an analysis of the topological structure and tolerance to failures and attacks of the Union for the Coordination of Transmission of Electricity power grid, by introducing a simple model that ignores physical characteristics of the grid, such as the impedance and capacity of lines. Chassin and Posse [2] propose a method for estimating system loss of load probability based only on the topological structure of the network and they test it on the North American eastern and western electric grids. Crucitti et al. [21] show that the Italian transmission grid is rather robust to most failures, but very vulnerable when the failures occur on the nodes with the highest betweenness, a measure of the importance of a node with respect to minimal paths of the graph. These and many other studies use pure topological metrics, neglecting the physics governing a power system. To fill these gaps, Arianos et al. [22] and Bompard et al. [23] combine a topological model with the dc load flow model and propose new metrics to provide an assessment of the system vulnerability, bringing into the model the concept of line flow limit. The strength of these graph-based approaches is to provide a global view of the system and identify emerging properties not necessarily designed in the system. On the negative side, important physical characteristics may be overlooked, especially when ignoring the dynamics and flows on the network and just considering the static topological picture.

\section{B. Sizing and Siting of Storage Systems}

Studies regarding the storage sizing problem typically aim at finding the optimal capacity at a fixed location, usually close to a wind farm or a large load. Chakraborty et al. [24] approach the problem by proposing a methodology for determining the sizing of energy storage system in order to reduce the total operational costs of two electric power systems with different number of thermal units and associated costs. In [25] and [26], batteries are combined with a wind farm, and the capacity is determined to reduce the difference between the expected and the actual production and to keep the injected power at a constant level, respectively. A novel perspective is presented in [27], where, using discrete Fourier transform to decompose the required balancing power into different periodic components, the optimal sizing of storage systems is determined in the time horizons over which they are most effective, according to their technologies. The storage siting problem is often connected with the previous one. Dvijotham et al. [28] develop a heuristic algorithm, which places storage at all buses with an unlimited power and energy capacities and then solves an optimization problem to restrict the number of devices, based on their activity at each node. Assuming a vertically integrated utility, Pandžić et al. [29] consider both the economic and technical aspects of the problem. By minimizing the sum of the generation costs and the daily investment cost in storage over a whole year, they identify the best storage locations based on the benefits that distributed storage units can perform by lessening congestions, and finally their optimal size is determined averaging over a year the daily maxima of stored energy and injected or extracted power. Ghofrani et al. [30] prove that distributed storage is to be preferred to a centralized one in order to better use the transmission capacity and more efficiently integrate the wind power in terms of profits.

\section{Power System AS A Network}

To perform an analysis of the siting and sizing of storage, one needs a sufficiently realistic model of the transmission grid. This is done by resorting to a dc load flow model and a weighted graph-based representation of the system.

\section{A. Linearized Power Systems' Model}

Power transmission systems operate in ac regime, where the complex power $\bar{S}_{i}$ that flows through a node $i$ is defined as follows:

$$
\bar{S}_{i}=P_{i}+j Q_{i}
$$


where $P$ and $Q$ are called real and reactive power, respectively. Since all quantities involved in an ac system have a sinusoidal waveform, solving a full ac power flow model means solving a system of nonlinear equations for each node $i$

$$
\left\{\begin{array}{l}
P_{i}=\sum_{j=1}^{N}\left|Y_{i j} V_{i} V_{j}\right| \cos \left(\theta_{i}-\theta_{j}-\gamma_{i j}\right) \\
Q_{i}=\sum_{j=1}^{N}\left|Y_{i j} V_{i} V_{j}\right| \sin \left(\theta_{i}-\theta_{j}-\gamma_{i j}\right)
\end{array}\right.
$$

where $V_{i}$ and $V_{j}$ are the voltage amplitudes at node $i$ and $j$, while $\theta_{i}, \theta_{j}$, and $\gamma_{i j}$ are the phases of voltages and line impedance, respectively. However, in a well-meshed grid, as it is the case for transmission grids and the IEEE-RTS 96 bus [31], some assumptions can be done to reduce the problem to a set of linear equations known as $D C$ power flow.

1) All voltages' magnitude can be considered constant and, as a consequence, normalized to 1 per unit (pu).

2) If resistances are at least an order of magnitude smaller than reactances, that is, the difference between reactances' and impedances' modules is very small, lines are assumed lossless, and only the reactances $x$ in pu are considered.

3) Differences between voltage angles are small.

4) All admittances are inductive.

5) Reactive power flows are ignored, as the power factor is very close to one.

In this way, the active power flowing between nodes $i$ and $j$ is given by

$$
f_{i j}=\frac{\theta_{i}-\theta_{j}}{x_{i j}} .
$$

The $\mathrm{dc}$ power flow is a useful approximation of the sinusoidal ac power flow equations and is frequently adopted, especially at the transmission level (see [22], [28], [29], [32]-[34]). For further illustration, the reader is referred to [35].

\section{B. Fundamentals of Network Theory}

A network is a collection of objects that are pairwise related. The objects are referred to as vertices or nodes, and the relationships are referred to as edges [36]. A power grid graph is a pair $(V, E)$ such that each element $v_{i} \in V$ is either a substation, transformer, or consuming unit of a physical power grid. There is an edge $e_{i, j}=\left(v_{i}, v_{j}\right)$ with $e_{i, j} \in E$ between two nodes if the elements represented by $v_{i}$ and $v_{j}$ are physically connected, by a power line. One can associate a weight to the edge representing a physical property (e.g., resistance and reactance). A weighted power grid graph is a power grid graph $G_{w}(V, E)$ with an additional function $w: E \rightarrow \mathbb{R}$ associating a real number to an edge representing a physical property of the physical cable represented by the edge [37]. The physical property can be the reactance of the line, taken in pu.

A path $P$ between two nodes is a subgraph of the form

$$
\begin{aligned}
& V(P)=\left\{v_{0}, v_{1}, \ldots, v_{l}\right\} \\
& E(P)=\left\{\left(v_{0}, v_{1}\right),\left(v_{1}, v_{2}\right), \ldots,\left(v_{l-1}, v_{l}\right)\right\}
\end{aligned}
$$

such that $V(P) \subseteq V$ and $E(P) \subseteq E$. The vertices $v_{0}$ and $v_{l}$ are the end-vertices or end-points of $P$ and $l=|E(P)|$ is the length of $P$, that is, the number of edges that the path $P$ contains [38]. According to this, given a graph $G$, the shortest path from $v_{i}$ to $v_{j}$ has the least number of edges among all paths for which $v_{i}$ and $v_{j}$ are the end-vertexes.

The betweenness $C_{b}(e)$ of an edge $e \in E$ is

$$
C_{b}(e)=\sum_{v_{i}, v_{j}} \sigma_{v_{i}, v_{j}}(e) / \sigma_{v_{i}, v_{j}}
$$

where $\sigma_{v_{i}, v_{j}}$ is the total number of shortest paths from node $v_{i}$ to node $v_{j}$ and $\sigma_{v_{i}, v_{j}}(e)$ is the number of those paths that pass through edge $e$. The concept of betweenness is useful to describe the importance of an edge (or a node) with respect to minimal paths. Vertices and edges that occur on many shortest paths between any given pair of vertices have higher betweenness than those that do not, that is, they have relatively higher importance within the graph [39]. The measures of betweenness, however, are based on the assumption that information flows only along the shortest paths, while in a power network, it is not always the case. According to the power flow equation (3), power flows along all available lines between two nodes, depending on the ratio of phase angles difference to reactance, and therefore other more precise measures have to be used.

Let $f_{\max }$ be the maximum flow from the node $v_{i}$ to the node $v_{j}$ during a single time step and let $f_{e}$ be the portion of maximum flow passing through the edge $e \in E$ of the network. The flow-based centrality index $C_{f}(e)$ is defined as [40]

$$
C_{f}(e)=\sum_{v_{i}} \sum_{v_{j}} f_{e}
$$

and, by dividing it by the total flow between all pairs of node, the above equation is normalized and expressed in percentage

$$
\widehat{C_{f}}(e)=\sum_{v_{i}} \sum_{v_{j}}\left[f_{e} / f_{\max }\right] \cdot 100 .
$$

The utilization index is defined to assess what percentage of the edge capacity is used. Let $f_{e}$ be the flow passing through the edge $e$ and let $c_{e}$ be its capacity, the utilization index $U(e)$ is defined as

$$
U(e)=\left[f_{e} / c_{e}\right] \cdot 100 .
$$

It is expressed as a percentage of the edge capacity. The edges are ranked according to the portion of flow that they carry or to how much they are exploited by resorting to (6) or (7), respectively.

\section{Model and Its Topological Representation}

In this paper, a modified version of the IEEE Reliability Test System 1996 (RTS-96) is used as a test case, where some renewable plants are added for a total capacity varying from $0 \%$ to $74 \%$ of winter total global load demands, lines' capacities are reduced to their $75 \%$, and some electrochemical storage systems are introduced. Parameters such as phase angle and reactance are included to achieve accurate results from the dc load flow, and the nodes are distinguished in four sets by assigning different properties. The most exploited 
lines are ranked by defining a utilization index, in order to determine what percentage of the line's capacity is used, and a multiperiod centrality analysis is provided, based on the index proposed in [40]. A set of batteries is added, whose size is determined according to the capacity of the connected lines. Different locations are chosen, by following three policies, in order to investigate their possible effects on the grid in terms of reduction of curtailment and relief of overloads and congestions.

The grid is topologically represented as a graph, and its main components are distinguished among four kinds of nodes and two types of edges. Generation plants $s_{i} \in S$ with $S \subset V$, that is, the sources set. This set is partitioned in two disjoint subsets renewable $R$ and conventional $P$; we consider only "nonprogrammable" sources in $R$, i.e., wind and photovoltaic (PV). Two subsets of $S$ are defined, such that $R \cup P=S$ and $R \cap P=0$. They inject power to be transmitted through the edges to the distribution substations. Each plant $s$ has upper $P_{s, \text { max }}$ and lower $P_{s, \text { min }}$ power generation limits that cannot be violated, depending on the technology. For renewable plants, the lower limit is assumed equal to zero, the upper one is the installed capacity $P_{r, \max }$, but the actual production is restricted by the power potentially available, $P_{r \text {,av }}$. Moreover, a marginal cost coefficient $c_{p}$ or $c_{r}$ (euro/MWh) is assigned to each plant according to its technology [41].

The load buses are sinks, $t_{i} \in T$ with $T \subset V$. They have only incoming flows, and their power demands have always to be satisfied. The power $l_{t}$ requested by the node $t$ can be valued through its assumed load in percentage $l_{t, \%}$ of the global system demand.

The storage nodes $b_{i} \in B$ with $B \subset V$ represent energy storage systems. Such nodes are allowed to act as generators or loads thus having both incoming and outgoing flows. In the first case, they behave as a device that charges, and in the second one, a device that discharges. Their main characteristics are as follows:

1) an upper limit of the state of charge $\mathrm{SoC}_{b, \max }$ expressed in terms of megawatt hour, that is, the energy capacity;

2) a lower limit of the state of charge $\mathrm{SoC}_{b, \min }$ in megawatt hour, that is

$$
\begin{aligned}
\mathrm{SoC}_{b, \text { min }} & =\left(100 \%-\mathrm{DoD}_{b}\right) \cdot \mathrm{SoC}_{b, \text { max }} \\
& =p_{\%} \cdot \mathrm{SoC}_{b, \text { max }}
\end{aligned}
$$

where $\mathrm{DoD}_{b}$ is the depth of discharge;

3) an upper limit of the rate of charge and discharge $\operatorname{ch}_{b, \max }$ in megawatts, that is, the power capacity;

4) an energy/rated power ratio $K_{b}=\mathrm{SoC}_{b, \max } / \mathrm{ch}_{b, \text { max }}$ in hours, that is, the duration that the battery can operate while delivering its rated output;

5) a charge and discharge efficiency, $\eta_{\mathrm{ch}}$ and $\eta_{\mathrm{dis}}$, respectively.

The time horizon considered is $24 \mathrm{~h}$, and each time step is $15 \mathrm{~min}$, thus resulting in 96 time slots per day. At the end of each time period $j$, the state of charge of the storage $b, \mathrm{SoC}_{b, j}$, is a function of its initial state $\mathrm{SoC}_{b, \text { in }}$ and of the incoming or outgoing flow during the period $\Delta j, \operatorname{ch}_{b, j}$, and $\operatorname{dis}_{b, j}$, respectively

$$
\operatorname{SoC}_{b, j}=\operatorname{SoC}_{b, \epsilon}+\operatorname{ch}_{b, j} \cdot \Delta j-\operatorname{dis}_{b, j} \cdot \Delta j
$$

where the relationship between the flows seen by the storage itself (i.e., internal flow), $\mathrm{ch}_{b, j}$, and $\operatorname{dis}_{b, j}$, and the one seen by the grid (i.e., external), $f(b, n)_{j}$, is governed by the following equations:

$$
\begin{aligned}
\operatorname{ch}_{b, j} & =f(b, n)_{j} \cdot \eta_{\mathrm{ch}} \\
\operatorname{dis}_{b, j} & =\frac{f(b, n)_{j}}{\eta_{\mathrm{dis}}} .
\end{aligned}
$$

Moreover, the state of charge has to be included between the upper and lower limits

$$
\mathrm{SoC}_{b, \text { min }} \leq \mathrm{SoC}_{b, j} \leq \mathrm{SoC}_{b, \max } .
$$

Finally, a set of inner nodes is defined as $n_{i} \in N$ with $N=V \backslash(S \cup T \cup B)$, and it includes all busbars.

The edges are distinguished in real and virtual. The real edges represent physical transmission lines. They have a weight equal to the line reactance in pu and their capacity is set equal the one of the line. Several components can be linked to the same inner node, by means of one or more switch connector in series with a circuit breaker. This group of elements is represented by a virtual edge that connects a source node (or a sink or a storage) to an inner one; a conventional weight of $10^{-4}$ is given. The introduction of virtual edges allows, indeed, characterizing every single node of the graph in a univocal way. The capacity of these edges is chosen great enough to drain out the upper power generation limit of the plant or to satisfy the power demand from the load.

The angle phase $\theta$ is a parameter assigned to all nodes in order to run a dc power flow, considering a base power $P_{b}$ of $100 \mathrm{MW}$.

\section{Sizing AND Siting of BATteries}

Given a power grid represented as a graph and an optimality measure, solving the sizing and siting problem consists in selecting a size and location on the network for storage such that the optimality measure scores best.

\section{A. Sizing}

The size of each storage added to the grid is determined by the capacity of the line it is connected to. It is assumed that the energy capacity of the battery to be set is sufficient to store energy for $1 \mathrm{~h}$ at the maximum flow allowed on the selected line. Furthermore, all storage systems are energy intensive applications, mainly oriented toward the shifting of energy flows over time. Therefore, a typical energy/rated power ratio value similar to those of NaS batteries is adopted (i.e., usually with values between six and eight) [42]. Moreover, the model assumes that a line $(u, v)$ has a capacity of $c(u, v)(\mathrm{MW})$ and the added storage has an energy capacity equal to $c(u, v)(\mathrm{MWh})$ and a power capacity equal to $c(u, v) / K$ (MW). As many devices as the lines whose capacities are saturated by a wind farm are added.

\section{B. Siting}

For each line, all utilization indexes can be ordered in descending order to obtain an overload duration curve that shows for how long the exploitation in percentage of a line is above a given level. In this way, a utilization index-based 
analysis helps to identify the most critical lines of the network during the day and classify them in a ranking to choose the best location for the storage systems.

Given a configuration of installed renewable plants, the quarters of an hour in which a line is loaded up to the $100 \%$ of its capacity on all days are counted; then, the average is calculated over all configurations. Four representative days are taken into consideration, that is, one per season. Thus, the maximum value that this parameter may obtain is 384 , considering 96 time steps per day as explained above. The ranking is compiled according to this average.

The lines can be distinguished between those linked to a wind farm and those that are not. The storage systems are located by following three different policies:

1) choosing a fixed number of buses connected to the most overloaded lines only among the ones linked to a wind farm;

2) choosing a fixed number of buses connected to the most overloaded lines among all the grid, in the order of the ranking;

3) choosing a fixed number of buses connected to the most overloaded lines in a random way.

The exploitation of batteries is considered as the optimality function to identify their best location. The time steps in which $\mathrm{SoC}_{b, j}>\mathrm{SoC}_{b, \text { min }}$ are counted, and the average over four days of the maximum energy stored and the average of the maximum power injected in or extracted from the battery are calculated.

\section{Minimization of Daily Production Costs}

To determine optimal size and site, the production costs over a determined time horizon are minimized, reducing the renewable power curtailment by means of electrochemical storage systems. A 24-h horizon and a 15-min resolution are taken into account. The state of the grid at the end of a time step $j$ represents the initial state of the next one, i.e., $j+1$.

When the available renewable production is too high to fulfill the constraint of balance or transmission capacity, then only a limited part of this is injected into the grid, while the one in excess is cut off. The amount of curtailed energy (MWh) from RESs can be valued as follows:

$$
\text { curtailment }=0.25 \sum_{j=0}^{95} \sum_{r \in R}\left(P_{r, j, \mathrm{av}}-P_{r, j}\right)
$$

where $P_{r, j, \text { av }}$ is the RES power potentially available at time step $j$. With the aim of minimizing the curtailment of wind or solar production, this solution is penalized with a high cost curt_cost.

\section{A. Objective Function}

The daily system cost to be minimized is defined as follows:

$$
\begin{aligned}
\min \sum_{j=0}^{95} & {\left[\sum_{p \in P} c_{p} \cdot P_{p, j}+\sum_{r \in R} c_{r} \cdot P_{r, j}\right.} \\
& \left.\quad+\text { curt_cost } \cdot \sum_{r \in R}\left(P_{r, j, \mathrm{av}}-P_{r, j}\right)\right]
\end{aligned}
$$

where $c_{p}$ and $c_{r}$ are the marginal cost coefficients [41]. The following linear constraints must be fulfilled.

1) Upper and lower power generation limits $\forall p \in P$

$$
P_{p, \min } \leq P_{p, j} \leq P_{p, \max } .
$$

2) Upper and lower power generation limits for RES plants $\forall r \in R$

$$
0 \leq P_{r, j} \leq P_{r, j, \text { av }} \leq P_{r, \max }
$$

3) Transmission line capacity $\forall(u, v) \in E$

$$
-c(u, v) \leq f(u, v)_{j} \leq c(u, v)
$$

where $f(u, v)_{j}$ is the flow from node $u$ to node $v$ at time step $j$.

4) Fulfillment of power demand $\forall t \in T$

$$
\sum_{v \in \Gamma(t)} f(v, t)_{j}=l_{t, j}
$$

where $\Gamma(t)$ is the set of all vertices linked to node $t$ through an edge.

5) Flow conservation $\forall n \in N$

$$
\sum_{(u, n) \in E} f(u, n)_{j}=\sum_{(n, z) \in E} f(n, z)_{j} .
$$

6) Balance between supply and demand

$$
\sum_{p \in P} P_{p, j}+\sum_{r \in R} P_{r, j}+\sum_{b \in B} f(b, n)_{j}=\sum_{t \in T} l_{t, j} .
$$

7) Power and energy flows $\forall b \in B$ if $f(b, n)>0$

$$
\begin{aligned}
& {\left[f(b, n)_{j} / \eta_{\text {dis }}\right]} \\
& \quad \leq \min \left(\left\{\operatorname{ch}_{b, \max }\right\},\left\{\left[\operatorname{SoC}_{b, j-1}-\operatorname{SoC}_{b, \min }\right] / \Delta j\right\}\right) .
\end{aligned}
$$

8) Power and energy flows $\forall b \in B$ if $f(b, n)<0$

$$
\begin{aligned}
& \left|f(b, n)_{j}\right| \eta_{\mathrm{ch}} \\
& \quad \leq \min \left(\left\{\operatorname{ch}_{b, \max }\right\},\left\{\left[\operatorname{SoC}_{b, \max }-\mathrm{SoC}_{b, j-1}\right] / \Delta j\right\}\right) .
\end{aligned}
$$

9) State of charge $\forall b \in B$

$$
p \% \cdot \operatorname{ch}_{b, \max } \cdot K \leq \mathrm{SoC}_{b, j} \leq \operatorname{ch}_{b, \max } \cdot K .
$$

10) Phase angle constraints

$$
\begin{aligned}
& \frac{-\pi}{2} \leq \theta_{v, j} \leq \frac{\pi}{2} \quad \forall v \in V \backslash s: \text { slack node } \\
& \theta_{s, j}=0 \quad s: \text { slack node. }
\end{aligned}
$$

This constraint is required to solve the system of $N$ equations and $N$ unknown bus voltage angles $P_{i}=$ $\sum_{j \in \Gamma(i)} f_{i j}=\sum_{j \in \Gamma(i)}\left(\theta_{i}-\theta_{j}\right) / x_{i j}$ obtained from dc load flow, as briefly explained in Section III-A.

\section{Test CAse And Results}

The model is applied on a slightly modified version of the IEEE RTS-96 [31], [43], while load profile data are coming from the Italian grid. Flows are computed on the test case by running a dc load flow and the optimal configuration is sought. 


\section{A. Test Case}

Starting from the IEEE RTS-96, renewable plants are added for a total capacity ranging from $0 \%$ to $74 \%$ of winter global load demands, in seven steps. The new suppliers are placed on the network according the following two policies:

1) cutting the $3,6,12$, or 15 longer transmission lines, that is, 1, 2, 4, or 5 per area, and adding a new internal node, a wind generation node, and a virtual edge;

2) adding a new PV solar generation node and a virtual edge to 9 or 12 load buses, that is, 3 or 4 per area.

Moreover, a minimal output power for each kind of generation plant is set by considering technical limits of different components, e.g., boiler and prime mover, and the capacities of the edges are reduced to their $75 \%$, since having a system operated in an $N-1$ security state [44] is not a goal of this paper, which focuses on the network behavior under critical conditions.

Renewable power injections are assumed to be known as a deterministic input that means having perfect forecasts. Changes due to forecast errors, while important in real-time operation, are less significant in the long-period horizon, that is, indeed, the context of the sizing and siting problem tackled by this paper. Renewable production profiles are estimated using historical wind and solar-PV generation data of two farms located in central Italy, whereas all load data are taken from [45], where the national load demands of the third Wednesday of each month of 2012 are recorded. Since the total installed production capacity in the RTS-96 amounts to 10.2 GW, which is considerably lower than the Italian value, all data have been adjusted. Four days, one per season, are considered in order to analyze different production-demand scenarios. For the detailed data, please refer to [46].

\section{B. Implementation}

To evaluate the configurations, the model and the objective function in (14) have been translated into a software program. It takes as input a file with the grid description and renewable production profiles. The software is developed in Java SE 7 and GNU Math Prog [47] in order to find the optimal solution for what is essentially a linear programming problem. The solution is found by means of the simplex method, which guarantees the results' optimality if the problem converges. For details about the implementation, the reader can refer to [48]. For these simulations, the configuration of the computer hardware used it: CPU Intel Core i5-2430M, $2.40 \mathrm{GHz}$, with 8 GB of RAM running Ubuntu 14.04 LTS 64 bit. The software guarantees the optimal solution with good performance in term of runtime and use of memory, around $82 \mathrm{~s}$ and $20 \mathrm{MB}$, respectively [46]. They both increase linearly with the number of nodes that means the software could be used for investigating the behavior of real grid with much more nodes, such as a part of the European HV network, by simply enhancing the hardware's computational capabilities.

\section{Results}

One of the goals is to identify the most stressed lines, in order to see where storage can be of relieve to the system.
TABLE I

RANKING OF THE LINES REACHING THEIR MAXIMUM CAPACITY BEFORE STORAGE INTEGRATION

\begin{tabular}{cccc}
\hline \hline Ranking & Line & Time steps & Renewable \\
\hline $\mathbf{1}$ & $132-133$ & 98 & \\
$\mathbf{2}$ & $117-178$ & 77 & $\checkmark$ \\
$\mathbf{3}$ & $95-177$ & 68 & $\checkmark$ \\
$\mathbf{4}$ & $96-180$ & 67 & $\checkmark$ \\
$\mathbf{5}$ & $141-179$ & 46 & $\checkmark$ \\
$\mathbf{6}$ & $156-157$ & 39 & \\
$\mathbf{7}$ & $108-109$ & 35 & \\
$\mathbf{8}$ & $152-173$ & 34 & $\checkmark$ \\
$\mathbf{9}$ & $153-176$ & 20 & $\checkmark$ \\
$\mathbf{1 0}$ & $165-170$ & 17 & $\checkmark$ \\
$\mathbf{1 0}$ & $128-172$ & 17 & $\checkmark$ \\
$\mathbf{1 1}$ & $154-156$ & 16 & \\
$\mathbf{1 2}$ & $146-150$ & 15 & \\
$\mathbf{1 3}$ & $130-132$ & 12 & \\
$\mathbf{1 4}$ & $117-118$ & 11 & \\
$\mathbf{1 5}$ & $143-179$ & 9 & \\
$\mathbf{1 5}$ & $158-169$ & 9 & \\
$\mathbf{1 6}$ & $98-102$ & 7 & \\
$\mathbf{1 7}$ & $118-122$ & 6 & \\
$\mathbf{1 8}$ & $129-175$ & 5 & $\checkmark$ \\
$\mathbf{1 9}$ & $113-170$ & 3 & \\
$\mathbf{2 0}$ & $119-140$ & 2 & \\
$\mathbf{2 1}$ & $93-177$ & 1 & \\
$\mathbf{2 1}$ & $99-100$ & 1 & \\
\hline Av. dur. & & 26 & \\
\hline \hline
\end{tabular}

TABLE II

STORAGE Systems' CHARACTERISTICS

\begin{tabular}{cccccc}
\hline $\begin{array}{c}\text { Line's capacity } \\
\text { MW }\end{array}$ & $\begin{array}{c}\mathbf{S o C}_{\mathbf{b}, \max } \\
\text { MWh }\end{array}$ & $\begin{array}{c}\mathbf{c h}_{\mathbf{b}, \max } \\
\text { MW }\end{array}$ & $\begin{array}{c}\mathbf{S o C}_{\mathbf{b}, \text { min }} \\
\text { MWh }\end{array}$ & $\begin{array}{c}\mathbf{S o C}_{\mathbf{b}, \mathbf{0}} \\
\text { MWh }\end{array}$ & $\#$ \\
\hline 131 & 131 & 19 & 27 & 27 & 4 \\
375 & 375 & 56 & 75 & 75 & 6 \\
\hline \hline
\end{tabular}

Table I provides a ranking of the edges according to the average number of time steps in which the edge reaches its capacity limit, that is, $U(e)_{j}=100$. Line $132-133$ is congested for 98 out of 384 quarters of hour, about $25 \%$ of the times, meaning that it represents a bottleneck of the grid. A $\checkmark$ sign indicates which lines are linked to renewable farms, in fact wind plants. PV solar plants are added, indeed, by the biggest loads and of a size such that their output is often not even enough for satisfying the local demand. Therefore, these plants do not cause lines' congestions.

Table II summarizes the characteristics of the storage added to the grid, by following the sizing policy explained in Section V-B. For both kinds of devices, the DoD and the charge/discharge efficiency are estimated to be equal to $80 \%$ and $87 \%$, respectively [49]. The energy/rated power ratios are 6.89 and $6.69 \mathrm{~h}$. Ten batteries are added by following three siting policies, referred to as "wind," "ranking," and "random" as described in Section V-B. The total power and energy capacities are $412 \mathrm{MW}$ and $2774 \mathrm{MWh}$, respectively.

Wind and solar productions are intermittent and volatile, so that the grid is not always able to totally absorb them due to transmission and operating constrains. Table III reports the curtailed energy for each supply-demand scenario and the sum over four days, before the introduction of batteries.

The curtailment mainly affects the wind farms. In the configuration, solar farms are involved for $40 \%$ of renewable installed capacity and for less than $2 \%$ of total curtailed energy, 


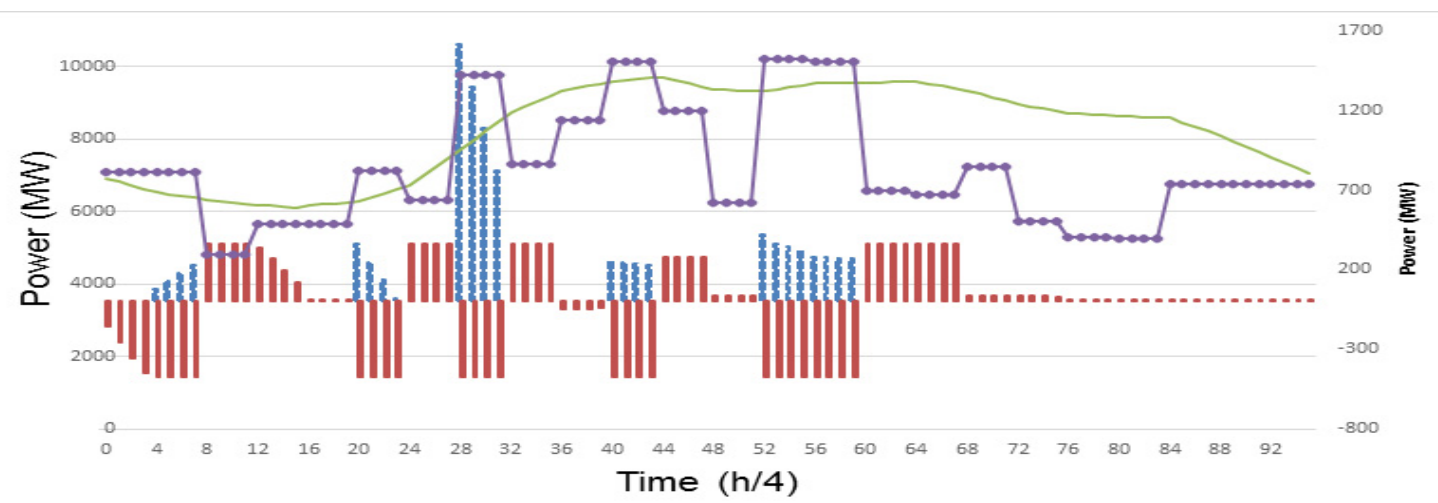

Curtailed power Batteries' flows — Load curve —-Adjusted renewable production

Fig. 1. Shifting of energy flows over time: batteries' optimal operation. The "adjusted renewable production" includes the minimum technical output of conventional generators, which cannot be reduced. One can see that the batteries charge (negative values of batteries' flows) when the load is lower than the available production, while they discharge (positive values) as soon as the load decreases. In this way, renewable energy is shifted over time and conventional generation is limited.

TABLE III

Curtailed Energy Before the Introduction of BATteries

\begin{tabular}{ccccccc}
\hline \hline & $\begin{array}{c}\text { Winter } \\
\text { MWh }\end{array}$ & $\begin{array}{c}\text { Spring } \\
\text { MWh }\end{array}$ & $\begin{array}{c}\text { Summer } \\
\text { MWh }\end{array}$ & $\begin{array}{c}\text { Autumn } \\
\text { MWh }\end{array}$ & $\begin{array}{c}\text { Total } \\
\text { MWh }\end{array}$ & $\begin{array}{c}\text { PV-solar } \\
\text { MWh }\end{array}$ \\
\hline $\mathbf{1 0 \%}$ & 0 & 0 & 0 & 550 & 550 & - \\
$\mathbf{2 0 \%}$ & 184 & 0 & 0 & 2755 & 2939 & - \\
$\mathbf{3 0 \%}$ & 2591 & 753 & 0 & 7784 & 11128 & - \\
$\mathbf{4 0 \%}$ & 4422 & 1258 & 81 & 11107 & 16867 & 66 \\
$\mathbf{5 0 \%}$ & 8250 & 2458 & 1448 & 18208 & 30363 & 418 \\
$\mathbf{6 6 \%}$ & 17003 & 4135 & 5241 & 26029 & 52408 & 579 \\
$\mathbf{7 4 \%}$ & 26635 & 5425 & 9001 & 32773 & 73834 & 668 \\
\hline \hline
\end{tabular}

TABLE IV

Curtailed EnERgy After the InTROduction OF BATteries B Y Following Three Policies

\begin{tabular}{lcccc}
\hline \hline & No batteries & Random & Ranking & Wind \\
\hline \multicolumn{5}{c}{$\mathbf{4 0 \%}$} \\
\hline Total (MWh) & 16867.4 & 12252.5 & 12252.5 & 12252.5 \\
Reduction \% & -27.36 & -27.36 & -27.36 \\
\hline \hline & $\mathbf{6 6 \%}$ \\
\hline Total (MWh) & 52408 & 40945.5 & 40966 & 41003.1 \\
Reduction \% & & -21.87 & -21.83 & -21.76 \\
\hline \hline
\end{tabular}

since they often do not inject power into the rest of the grid.

With low levels of renewable penetration, batteries would be scarcely used. Therefore, the possible effects of these devices are investigated with the renewable installed capacity equal to $40 \%$ and $66 \%$ of the winter global load demands.

An example of batteries' optimal operation to minimize the daily production costs is shown in Fig. 1. The adjusted renewable production includes the minimum technical output of conventional generators. Batteries' flows are positive during discharging and negative during charging (right $y$-axis). As one can see, the devices are charged when the available production is higher than load demand and discharge as soon as possible to limit the supply from conventional plants.

Different locations do not significantly affect the reduction in energy curtailment that amounts to $-27.36 \%$ and $-21.87 \%$, as evident from Table IV. The small differences in the second scenario are attributed to local transmission congestions that do not allow the charge of a battery, resulting in a nonoptimal operation. Moreover, Table $\mathrm{V}$ reports the
TABLE V

SUMmary TABLE ABOUT BATTERIES' EXPLOITATION WHEN "WIND" POLICY IS APPLIED FOR SITING

\begin{tabular}{|c|c|c|c|c|c|c|c|c|}
\hline \multirow[t]{3}{*}{ Type } & \multicolumn{4}{|c|}{$40 \%$} & \multicolumn{4}{|c|}{$66 \%$} \\
\hline & \multirow[t]{2}{*}{ h/4 } & \multicolumn{2}{|c|}{$\mathbf{S o C}_{\text {av }}$} & \multirow{2}{*}{$\begin{array}{r}\mathbf{c h}_{\text {av }} \\
\text { MW }\end{array}$} & \multirow[t]{2}{*}{$h / 4$} & \multicolumn{2}{|c|}{ SoC $_{\text {av }}$} & \multirow{2}{*}{$\begin{array}{l}\mathbf{c h}_{\text {av }} \\
\text { MW }\end{array}$} \\
\hline & & MWh & $\%$ & & & MWh & $\%$ & \\
\hline $131 / 19$ & 107 & 71.5 & 54.5 & 16.7 & 245 & 97.8 & 74.7 & 19.0 \\
\hline $375 / 56$ & 81 & 203.6 & 54.3 & 51.5 & 233 & 265.3 & 70.8 & 56.0 \\
\hline
\end{tabular}

batteries' exploitation, as explained in Section V-B. Without affecting the validity of the overall considerations, only the Wind siting policy is reported in Table V. The batteries' utilization considerably increases with the percentage of RES installed in terms of time steps and stored energy. However, the energy capacity is rarely fully exploited that results in a maximum $\mathrm{SoC}_{\mathrm{av}}$ of $74.7 \%$ and $70.7 \%$ for the two types of devices.

Considering lines' congestions, the storage introduction causes a redispatch of power flows, which changes with different siting policies [50], but the average overload duration among all lines remains almost the same. With a further examination, one can see examples of transmission congestions' resolution. A daily chronological power flow of the most stressed line is shown in Fig. 2, before and after the introduction of storage. This power line (edge in the model) is located in a strategical position between an area with an excess of conventional production and one with many loads. One can see that without batteries, the line is overload in several time steps (i.e., 32-35, 60-67, and 76-83). After the introduction of storage systems, thanks to their discharge (positive values), the congestions are avoided in $32-35$ and $60-67$, when the flow is significantly reduced, using previously stored energy. However, the batteries keep discharging and, therefore, once the line has to face another contingency (76-83), not all of them can discharge at their maximum power capacity, limiting the positive effects. Overall, in this case, the overload is reduced from 20 time steps to 4 , that is a reduction of $80 \%$.

Finally, the centrality index defined in (6) is applied on the multistage time horizon, using a weighted mean approach with 


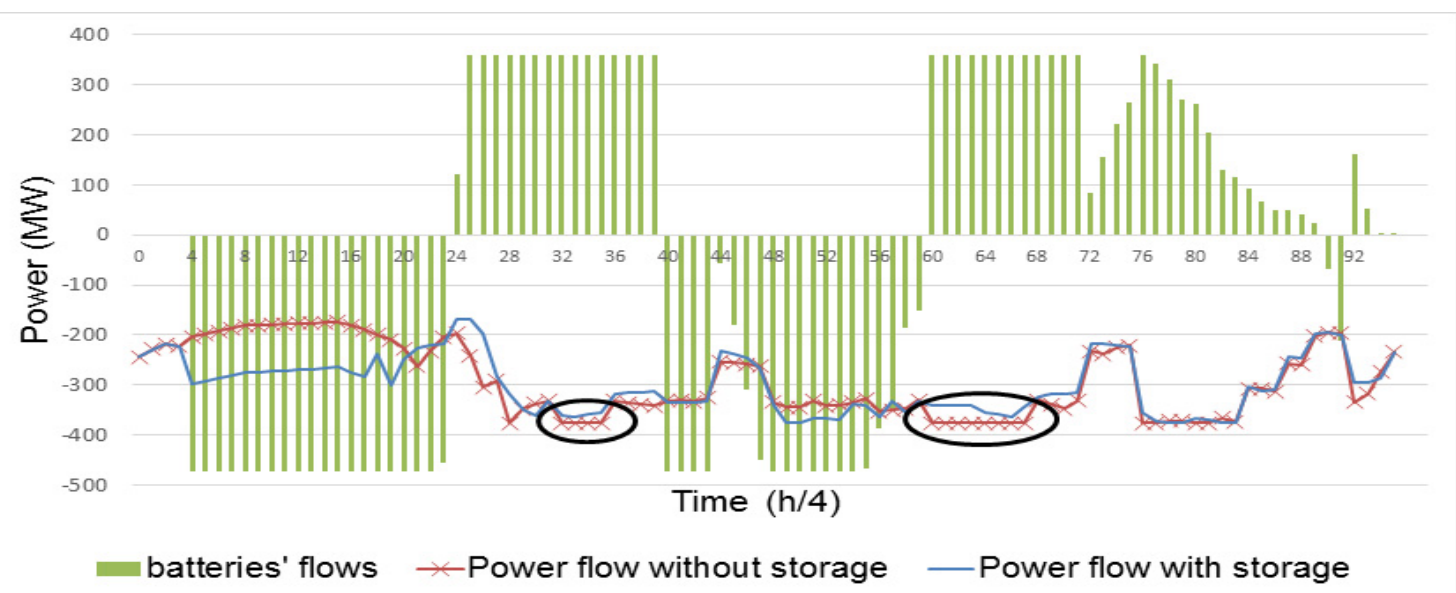

Fig. 2. Transmission congestion: comparison between chronological power flows without and with storage on the most stressed line in Summer, with 66\% of RES penetration and storage located according to "wind" policy. The number of time step in which the line reaches its flow limit ( \pm 375 MW) is significantly reduced.

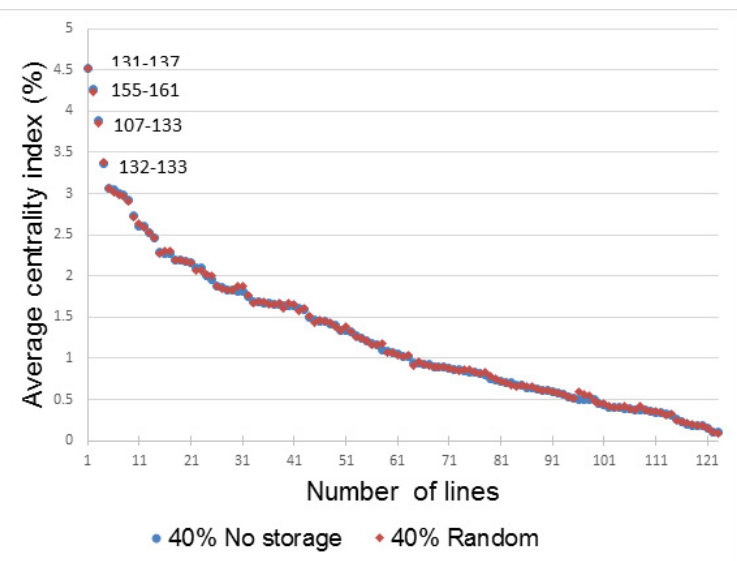

Fig. 3. Centrality index trend: from no storage to introduction of batteries. This index, which is flow-based but does not take into account lines' flow limits, is influenced by neither the sizing nor the siting of storage.

total load demands as weights, to perform a centrality analysis. The total flow on the grid is far greater than the flow on a line; therefore, the flow-based centrality index is not significantly affected by the introduction of storage, whatever siting policy is followed, as expected. The RES production influences the centrality index instead: for lines carrying mainly energy from conventional plants, this parameter tends to decrease as the renewable capacity increases, vice-versa it decreases for the edges linked to wind farms [46]. A comparison of overall trend of average centrality index between a scenario with $40 \%$ of RES, before and after introduction of batteries, is shown in Fig. 3. There are four edges with a high value, that is, this small portion of lines carries a significant part of the global flow (around 16\%), followed by an almost linear trend. These edges transmit the production of plants with small marginal costs, e.g., nuclear and hydroelectric ones; from the upper to the lower part of each area, there are more expensive plants and more loads. This result shows how good and optimized is the IEEE RTS-96 model, although real networks might not have such a smooth behavior and show key bottlenecks [51].

\section{CONCLUSION}

The need to increase the amount of renewable sources in high-voltage networks is changing their traditional operational conditions. Growing amount of rapid and unpredictable power has to be dispatched, while the balance and reliability of the system have to be guaranteed. Such trend poses the necessity to take into account not only the topological aspects of the network but also physical properties and laws governing power flows.

In this paper, large-scale storage systems are introduced in a transmission network with high RES penetration, and their appropriate size and location are sought in order to foresee a smart power grid. By considering configuration with the introduction of storage devices into transmission grids, the problem is mathematically formulated as the minimization of the production costs over a 24-h horizon. Taking four different supply-demand scenarios and RES power capacities, ten batteries, and a modified version of IEEE-RTS 96, three siting policies (i.e., random, ranking, and wind) are followed. Representing the network as a weighted graph, by including the physical characteristics of its main components, is helpful in having a valid model of a realistic infrastructure, without needing a very precise level of details. Moreover, the present method applies metrics based on real flows, instead of on static topological considerations (e.g., shortest paths on graphs) on a multiperiod scenario and it shows good scalability that means larger network samples could be analyzed.

The introduction of batteries allows the grid to store energy when the renewable availability is excessive and use them to limit the supply from conventional plants at a later time, during high-demand periods. Even if detailed economic considerations are out of the scope of this paper, it shows how proper sizing (and to a smaller extent siting) of batteries can be a key step toward a more flexible and smarter use of transmission grids, as it is required by the increasing exploitation of renewables.

The analysis evidences that, on the one hand, the different policies of siting have no significant influence on the average overload duration of the lines, and on the other hand, the 
introduction of storage appears to considerably reduce the congestion of critical corridors. Somewhat unexpectedly, the proposed method shows that placing a storage close to a wind farm does not automatically imply that the major contributions in terms of relief of congestions are gained on the lines linked to the renewable nodes itself. Instead, the major positive effects are achieved on critical junctions, even if no storage is located to their end-nodes. This result is important both from a technical and an economical point of view. The action of the storage can help while a permanent mitigation measure, such as generation redistribution or startup of a fast unit, takes effect or, in the best case, can lower the flow on the line for a time frame long enough to overcome the contingency. The mentioned mitigation measures are, indeed, expensive, since they would usually involve generators with fast ramp rates, such as gas or oil-fired facilities.

The centrality analysis shows that this index is not significantly affected by neither the sizing nor the siting of storage. This result underlines that an approach that does not take into account flow limits might be weak when investigating crucial aspects of power systems concerning congestion and power flows' distribution.

Based on this paper, further research is on-going to understand to what extent the advantages of introducing batteries can be enhanced. Different siting policies are under consideration, together with an economic evaluation of the benefits when considering market-related requirements.

\section{REFERENCES}

[1] M. Rosas-Casals, S. Valverde, and R. Solé, "Topological vulnerability of the european power grid under errors and attacks," Int. J. Bifurcation Chaos, vol. 17, no. 7, pp. 2465-2475, 2007.

[2] D. P. Chassin and C. Posse, "Evaluating North American electric grid reliability using the Barabási-Albert network model," Phys. A, Statist. Mech. Appl., vol. 355, nos. 2-4, pp. 667-677, Sep. 2005.

[3] G. Chen, Z. Y. Dong, D. J. Hill, and G. H. Zhang, "An improved model for structural vulnerability analysis of power networks," Phys. A, Statist. Mech. Appl., vol. 388, no. 19, pp. 4259-4266, Oct. 2009.

[4] A. Dwivedi, X. Yu, and P. Sokolowski, "Identifying vulnerable lines in a power network using complex network theory," in Proc. IEEE Int. Symp. Ind. Electron. (ISIE), Jul. 2009, pp. 18-23.

[5] C. D. Brummitt, P. D. H. Hines, I. Dobson, C. Moore, and R. M. D'Souza, "Transdisciplinary electric power grid science," Proc. Nat. Acad. Sci. USA, vol. 110, no. 30, p. 12159, 2013.

[6] M. Rosas-Casals et al., "Knowing power grids and understanding complexity science," Int. J. Critical Infrastruct., vol. 11, no. 1, pp. 4-14, 2015.

[7] L. Furby, P. Slovic, B. Fischhoff, and R. Gregory, "Public perceptions of electric power transmission lines," J. Environ. Psychol., vol. 8, no. 1, pp. 19-43, Mar. 1988.

[8] M. Eddy. (Feb. 2014). Germany's clead-energy plan faces resistance to power lines. The New York Times. [Online]. Available: http://www.nytimes.com

[9] G. A. Pagani and M. Aiello, "From the grid to the smart grid, topologically," Phys. A, Statist. Mech. Appl., vol. 449, pp. 160-175, May 2016. [Online]. Available: http://www.sciencedirect.com/science/article/ pii/S0378437115011085

[10] S. Barsali, R. Giglioli, M. Giuntoli, G. Lutzemberger, and D. Poli, "Control strategies and real time operation of storage systems integrated with MV photovoltaic plants," in Proc. IEEE 15th Int. Conf. Environ. Elect. Eng. (EEEIC), Jun. 2015, pp. 243-248.

[11] Europe 2020: A Strategy for Smart, Sustainable and Inclusive Growth, vol. 3, Eur. Commission, Brussels, Belgium, 2010.

[12] President Obama Announces New Actions to Bring Renewable Energy and Energy Efficiency to Households Across the Country, Office Press Secretary, Washington, DC, USA, 2015.
[13] S. Barsali, M. Ceraolo, R. Giglioli, and D. Poli, "Storage applications for smartgrids," Elect. Power Syst. Res., vol. 120, pp. 109-117, Mar. 2015. [Online]. Available: http://www.sciencedirect.com/science/article/ pii/S0378779614002843

[14] A. Del Rosso and S. Eckroad, "Application of storage technology for transmission system support: Interim report," EPRI, Palo Alto, CA, USA, Tech. Rep. 1025418, 2012.

[15] D. Rastler, "Electric energy storage technology options a primer on applications, costs \& benefits," EPRI, Palo Alto, CA, USA, Tech. Rep. 1022261, 2010.

[16] M. Ceraolo, G. Lutzemberger, and D. Poli, "Aging evaluation of high power lithium cells subjected to micro-cycles," J. Energy Storage, vol. 6 , pp. 116-124, May 2016. [Online]. Available: http://www.sciencedirect. com/science/article/pii/S2352152X16300482

[17] D. Poli, A. di Donato, and G. Lutzemberger, "Experiences in modeling and simulation of hydrogen fuel-cell based propulsion systems," SAE Tech. Paper 2009-24-0084, 2009. [Online]. Available: http://dx.doi.org/10.4271/2009-24-0084

[18] M. Ceraolo, G. Lutzemberger, M. Funaioli, L. Sani, and D. Poli, "Electrical storage for the enhancement of energy and cost efficiency of urban railroad systems," in Proc. 2nd Int. Conf. Railway Technol., vol. 104, no. 244, pp. 62-78, Apr. 2014.

[19] G. A. Pagani and M. Aiello, "The power grid as a complex network: A survey," Phys. A, Statist. Mech. Appl., vol. 392, no. 11, pp. 2688-2700, 2013.

[20] G. A. Pagani and M. Aiello, "Towards decentralization: A topological investigation of the medium and low voltage grids," IEEE Trans. Smart Grid, vol. 2, no. 3, pp. 538-547, Sep. 2011.

[21] P. Crucitti, V. Latora, and M. Marchiori, "A topological analysis of the Italian electric power grid," Phys. A, Statist. Mech. Appl., vol. 338, nos. 1-2, pp. 92-97, Jul. 2004.

[22] S. Arianos, E. Bompard, A. Carbone, and F. Xue, "Power grid vulnerability: A complex network approach," Chaos, Interdiscipl. J. Nonlinear Sci., vol. 19, no. 1, p. 013119, 2009.

[23] E. Bompard, R. Napoli, and F. Xue, "Extended topological approach for the assessment of structural vulnerability in transmission networks," IET Generat., Transmiss. Distrib., vol. 4, no. 6, pp. 716-724, Jun. 2010.

[24] S. Chakraborty, T. Senjyu, H. Toyama, A. Y. Saber, and T. Funabashi, "Determination methodology for optimising the energy storage size for power system," IET Generat., Transmiss. Distrib., vol. 3, no. 11, pp. 987-999, Nov. 2009.

[25] T. K. A. Brekken, A. Yokochi, A. von Jouanne, Z. Z. Yen, H. M. Hapke and D. A. Halamay, "Optimal energy storage sizing and control for wind power applications," IEEE Trans. Sustain. Energy, vol. 2, no. 1, pp. 69-77, Jan. 2011

[26] X. Y. Wang, D. M. Vilathgamuwa, and S. S. Choi, "Determination of battery storage capacity in energy buffer for wind farm," IEEE Trans. Energy Convers., vol. 23, no. 3, pp. 868-878, Sep. 2008.

[27] Y. Makarov, P. Du, M. C. W. Kintner-Meyer, C. Jin, and H. Illian, "Sizing energy storage to accommodate high penetration of variable energy resources," IEEE Trans. Sustain. Energy, vol. 3, no. 1 pp. 34-40, Jan. 2012.

[28] K. Dvijotham, S. Backhaus, and M. Chertkov. (Jul. 2011). "Operationsbased planning for placement and sizing of energy storage in a grid with a high penetration of renewables." [Online]. Available: https://arxiv.org/abs/1107.1382

[29] H. Pandžić, Y. Wang, T. Qiu, Y. Dvorkin, and D. S. Kirschen, "Nearoptimal method for siting and sizing of distributed storage in a transmission network," IEEE Trans. Power Syst., vol. 30, no. 5, pp. 2288-2300, Sep. 2014.

[30] M. Ghofrani, A. Arabali, M. Etezadi-Amoli, and M. S. Fadali, "A framework for optimal placement of energy storage units within a power system with high wind penetration," IEEE Trans. Sustain. Energy, vol. 4, no. 2, pp. 434-442, Apr. 2013.

[31] P. Wong et al., "The IEEE reliability test system-1996. A report prepared by the reliability test system task force of the application of probability methods subcommittee," IEEE Trans. Power Syst., vol. 14, no. 3, pp. 1010-1020, Aug. 1999.

[32] S. Pahwa, C. Scoglio, and N. Schulz. (Dec. 2012). Topological Analysis and Mitigation Strategies for Cascading Failures in Power Grid Networks. [Online]. Available: http://arxiv.org/abs/1212.5620

[33] I. C. Costa, M. A. Da Rosa, L. M. Carvalho, F. J. Soares, L. Bremermann, and V. Miranda, "Probabilistic analysis of stationary batteries performance to deal with renewable variability," in Proc. Int. Conf. Probabilistic Methods Appl. Power Syst. (PMAPS), Jul. 2014, pp. 1-6. 
[34] K. Dvijotham, M. Chertkov, and S. Backhaus, "Storage sizing and placement through operational and uncertainty-aware simulations," in Proc. 47th Hawaii Int. Conf. Syst. Sci. (HICSS), Jan. 2014, pp. 2408-2416.

[35] J. D. D. Glover and M. S. Sarma, Power System Analysis and Design, 3rd ed. Pacific Grove, CA, USA: Brooks/Cole Publishing Co., 2001.

[36] M. Newman, Networks: An Introduction. New York, NY, USA: Oxford Univ. Press, 2010.

[37] G. A. Pagani, "From the grid to the smart grid, topologically," Ph.D. dissertation, Univ. Groningen, Groningen, The Netherlands, 2014. [Online]. Available: http://www.rug.nl/

[38] B. Bollobás, Modern Graph Theory, 1st ed. New York, NY, USA: Springer, 1998.

[39] L. C. Freeman, "A set of measures of centrality based on betweenness," Sociometry, vol. 40, no. 1, pp. 35-41, Mar. 1977.

[40] A. Dwivedi, X. Yu, and P. Sokolowski, "Analyzing power network vulnerability with maximum flow based centrality approach," in Proc. 8th IEEE Int. Conf. Ind. Inform. (INDIN), Jul. 2010, pp. 336-341.

[41] A. O'Sullivan and S. Sheffrin, Economics: Principles Action (Prentice Hall Science/Social Studies). Englewood Cliffs, NJ, USA: Prentice-Hall, 2007.

[42] M. Beaudin, H. Zareipour, A. Schellenberglabe, and W. Rosehart, "Energy storage for mitigating the variability of renewable electricity sources: An updated review," Energy Sustain. Develop., vol. 14, no. 4, pp. 302-314, Dec. 2010.

[43] M. Giuntoli, P. Pelacchi, and D. Poli, "On the use of simplified reactive power flow equations for purposes of fast reliability assessment," in Proc. EUROCON, Jul. 2013, pp. 992-997.

[44] X. Wang, Y. Song, and M. Irving, "Load flow analysis," in Modern Power Systems Analysis. Boston, MA, USA: Springer, 2008, pp. $71-128$

[45] Terna. (2012). Dati Statistici Sull'energia Elettrica in Italia. [Online]. Available: http://www.terna.it/archiviodocumenti.aspx

[46] L. Fiorini, "The introduction of storage in HV grids: Optimal use of renewable sources," M.S. thesis, Dept. DESTEC, Univ. Pisa, Pisa, Italy, 2015. [Online]. Available: http://www.cs.rug.nl/ /tesi/fiorini.pdf

[47] Wikibooks. GLPK/GMPL (MathProg), accessed on Mar. 2015. [Online]. Available: https://en.wikibooks.org/wiki/GLPK/GMPL_(MathProg)

[48] E. Vineti, "Strumenti di ottimizzazione per le reti elettriche," Bachelor thesis, Univ. Modena Reggio Emilia, Modena, Italy, 2015

[49] A. C. Tortora, E. Senatore, L. Apicella, and R. Polito, "Sistemi di accumulo di energia elettrochimici per la gestione efficiente delle fonti rinnovabili non programmabili," L'Energia Elett., vol. 91, no. 4, pp. 35-46, Jul./Aug. 2014.

[50] M. Giuntoli and D. Poli, "On the possible use of probabilistic techniques for purposes of short-term load dispatching," Int. Rev. Electr. Eng., vol. 8, no. 4, pp. 1243-1252, 2013.

[51] V. Rosato, S. Bologna, and F. Tiriticco, "Topological properties of high-voltage electrical transmission networks," Elect. Power Syst. Res., vol. 77, no. 2, pp. 99-105, Feb. 2007.

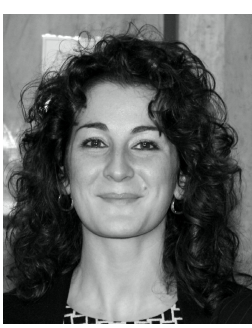

Laura Fiorini received the B.Sc. and M.Sc. degrees in electrical engineering from the University of Pisa, Pisa, Italy, in 2013 and 2015, respectively. She is currently pursuing the Ph.D. degree with the Distributed Systems Group, Faculty of Mathematics and Natural Sciences, University of Groningen, Groningen, The Netherlands.

She was a Research Assistant at the University of Groningen. Her current research interests include smart grids, integration and optimal operation of energy systems, and renewable energy.

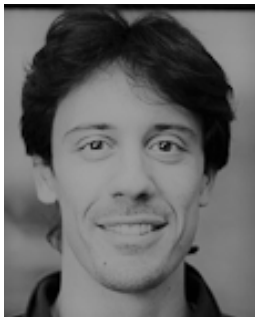

Giuliano Andrea Pagani (S'10-M'15) received the B.Sc. and M.Sc. degrees in computer engineering from the University of Parma, Parma, Italy, in 2004 and 2006, respectively, the M.S. degree in corporate management from the MIP Polytechnic University of Milan, Milan, Italy, in 2009, and the Ph.D. degree in computer science from the University of Groningen, Groningen, The Netherlands, in 2014.

He was a Post-Doctoral Researcher at the University of Groningen and the IBM Research T. J. Watson, Yorktown Heights, NY, USA, from 2014 to 2015, and was with the Dutch DSO Alliander N.V., Arnhem, The Netherlands, from 2015 to 2016 . He is currently a Data Scientist with the Royal Netherlands Meteorological Institute, De Bilt, The Netherlands, and is an external Post-Doctoral Researcher with the University of Groningen. His current research interests include distributed energy generation with a focus on complex network analysis, graph theory, smart grid technology, and renewable energy.

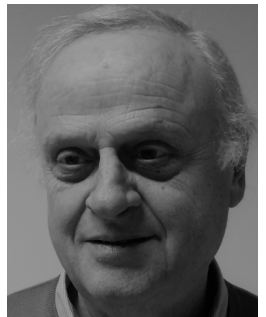

Paolo Pelacchi received the master's degree in electrical engineering from the University of Pisa, Pisa, Italy, in 1976.

In 1979, he joined the Electrical Research Center of ENEL, Milan, Italy, the national electricity utility before the market deployment. Since 1983, he has been a member of the Energy Department with the University of Pisa, first as a Researcher, then as an Associate Professor in 1992, and has been a Full Professor of Electric Power Systems since 2000. His current research interests include transmission and distributed generation problems in an electricity market deregulation context.

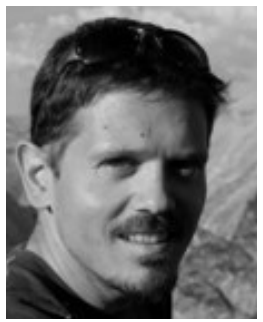

Davide Poli (M'13) received the master's and Ph.D degrees in electrical engineering from the University of Pisa, Pisa, Italy, in 1997 and 2002, respectively.

$\mathrm{He}$ was an Associate Professor with the Energy Section of DESTEC, Pisa, regards smart grids and power systems deregulation, in particular new simulating tools to assess the impact of energy and ancillary services markets on system reliability, security, and efficiency.

Dr. Poli is a member of CIGRE WG C6.20 (Impact of electric vehicles on the power system).

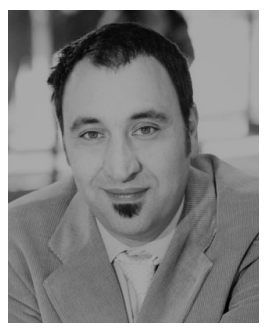

Marco Aiello (S'97--M'02--SM'12) received the $\mathrm{Ph} . \mathrm{D}$. degree from the University of Amsterdam, Amsterdam, The Netherlands, and the M.Sc. cum laude degree in engineering from the University of Rome La Sapienza, Rome, Italy.

He was a Lise Meitner Fellow at the Technical University of Vienna, Vienna, Austria, from which he received the Habilitation, and was an Assistant Professor at the University of Trento, Trento, Italy $\mathrm{He}$ is currently a Full Professor of Distributed Systems with the University of Groningen, Groningen, The Netherlands, and is the Head of the Distributed Systems Unit. His current research interests include smart energy systems, smart grids, and complex networks. 